\title{
Carbon Nanofibers Have IgE Adjuvant Capacity but Are Less Potent Than Nanotubes in Promoting Allergic Airway Responses
}

\author{
Unni Cecilie Nygaard, ${ }^{1}$ Mari Samuelsen, ${ }^{1}$ Calin Daniel Marioara, ${ }^{2}$ and Martinus Løvik ${ }^{1,3}$ \\ ${ }^{1}$ Division of Environmental Medicine, Norwegian Institute of Public Health, P.O. Box 4404 Nydalen, 0403 Oslo, Norway \\ ${ }^{2}$ SINTEF Materials and Chemistry, Høyskoleringen 5, 7465 Trondheim, Norway \\ ${ }^{3}$ Department of Cancer Research and Molecular Medicine, Faculty of Medicine, Norwegian University of Science and Technology, \\ Olav Kyrres Gate 9, 7489 Trondheim, Norway
}

Correspondence should be addressed to Unni Cecilie Nygaard; unni.cecilie.nygaard@fhi.no

Received 31 May 2013; Revised 11 July 2013; Accepted 12 July 2013

Academic Editor: Alexandre Paula Rogerio

Copyright (C) 2013 Unni Cecilie Nygaard et al. This is an open access article distributed under the Creative Commons Attribution License, which permits unrestricted use, distribution, and reproduction in any medium, provided the original work is properly cited.

\begin{abstract}
There is a growing concern for the possible health impact of nanoparticles. The main objective of this study was to investigate the allergy-promoting capacity of four different carbon nanofiber (CNF) samples in an injection and an airway mouse model of allergy. Secondly, the potency of the CNF was compared to the previously reported allergy-promoting capacity of carbon nanotubes (CNT) in the airway model. Ultrafine carbon black particles ( $\mathrm{UCCBP}$ ) were used as a positive control. Particles were given together with the allergen ovalbumin (OVA) either by subcutaneous injection into the footpad or intranasally to BALB/cA mice. After allergen booster, OVA-specific IgE, IgG1, and IgG2a in serum were measured. In the airway model, inflammation was determined as influx of inflammatory cells (eosinophils, neutrophils, lymphocytes, and macrophages) and by mediators (MCP-1 and TNF- $\alpha$ present in bronchoalveolar fluid (BALF)). CNF and CNT both increased OVA-specific IgE levels in the two models, but in the airway model, the CNT gave a significantly stronger IgE response than the CNF. Furthermore, the CNT and not the CNF promoted eosinophil lung inflammation. Our data therefore suggest that nanotube-associated properties are particularly potent in promoting allergic responses.
\end{abstract}

\section{Introduction}

Allergic airway diseases are characterized by eosinophil and lymphocyte lung inflammation, as well as allergen-specific IgE in serum. Ultrafine particles present in ambient air have, in animal models and in humans, been demonstrated to modulate airway inflammations and promote allergic responses in the lung [1-4]. Carbon nanotubes (CNT) are particles applied in an increasing number of consumer products and are also incidental components in indoor air pollution $[5,6]$. Carbon nanofibers (CNF) are useful in many of the same applications as $\mathrm{CNT}$, such as $\mathrm{Li}$-ion batteries and polymer nanocomposites [7]. In parallel to the increasing manufacture of CNT and $\mathrm{CNF}$, there is a growing concern for the health impact of these nanoparticles in occupational workers and consumers in general, also in relation to allergy. Inhalation studies in rodents suggest that CNT may induce toxic effects like transient inflammation, fibrosis, and granuloma formation [8], and mice with a preexisting allergic inflammation have been reported to be particularly susceptible [9]. Further, single-walled (sw) and multiwalled (mw) CNT have been reported to increase allergen-specific IgE levels, eosinophil airway inflammation, and Th2-associated cytokine responses in mice models $[2,10,11]$. In support, Park and coworkers demonstrated increased total IgE, Th2-associated cytokine, and $\mathrm{B}$ cell levels in mice after a single intratracheal exposure to mwCNT [12]. However, little is known about the characteristics of CNT responsible for the allergy-promoting effect. The possible allergy-promoting properties of CNF have not been investigated.

The main objective of this study was to investigate the allergy-promoting capacity of four different CNF samples in an injection and an airway mouse model of allergy. Secondly, the potency of the CNF was compared to the 
previously reported allergy-promoting capacity of CNT and ultrafine carbon black particles (ufCBP) in the airway model. Knowledge on which physicochemical characteristics of the particles are important for their biological effects is essential to enable manufacturing of particles with low toxicity. We, therefore, explored the importance of major physicochemical properties of carbon nanoparticles on their capacity to modulate allergy, by comparing the allergy-promoting capacity of samples having different physicochemical properties. Four samples of well-characterized, qualitatively different CNF produced in the same pilot plant were tested in both mouse models (CNF A, B, C, and D [7]). By deliberate use of different conditions and purification steps during the CNF manufacturing process, four samples with different particle properties such as fiber width and length, open versus closed channels, metal impurity content, relative surface area, presence of structural defects, and relative amount of fibers to other graphitic material were produced. Samples of swCNT, mwCNT, and "spherical" ufCBP (Printex90), previously shown to promote allergic responses in the two mouse models used [2], were also included in the airway model. The allergy parameters allergen-specific IgE levels in serum and eosinophil lung inflammation were measured. Allergenspecific IgG2a antibodies and the presence of inflammatory cells, MCP-1, and TNF- $\alpha$ in bronchoalveolar lavage fluid (BALF) were also determined, to reflect Th1- and general inflammatory responses.

\section{Material and Methods}

2.1. Animals. Female inbred BALB/cAnNCrl mice (Charles River, Sulzfeld, Germany) were 6-7 weeks old upon arrival and were acclimatised for one week. Four animals were housed per cage in Innorack IVC (Innovive Inc., CA, USA), containing Nestpaks filled with aspen $4 \mathrm{HK}$ bedding (Datesand Ltd, Manchester, UK). The mice were exposed to a $12 \mathrm{hr} / 12 \mathrm{hr}$ light/dark cycle (30-60 lux in cages), regulated room temperature $\left(20 \pm 2^{\circ} \mathrm{C}\right)$, and $40-60 \%$ relative humidity. Pelleted food (RM1, SDS, Essex, UK) and tap water were provided ad libitum. The experiments were performed in conformity with the laws and regulations for experiments with live animals in Norway and were approved by the Norwegian Experimental Animal Board under the Ministry of Agriculture (FOTS ID numbers 678 and 1005).

2.2. Particle Source and Characteristics. Four qualitatively different batches of carbon nanofibers (CNF A, B, C and D) were kindly provided by Statoil and Elkem Carbon AS (Kristiansand, Norway). The particles were produced from natural gas in a catalytic chemical vapor deposition (CVD) process and processed as reported [11]. The swCNT and mwCNT, also produced by CVD method, were obtained from Sigma-Aldrich (St. Louis, MO, USA, Cat. numbers 636797 and 636487, resp.). Ultrafine carbon black particles (ufCBP, Printex90), kindly provided by Degussa (Köln, Germany), were included as a positive reference particle for adjuvant activity in mice $[1,2]$. All particle samples were characterized by transmission electron microscopy (TEM), and the specific surface area was determined by nitrogen adsorption using the Brunauer, Emmet, and Teller method (BET). The endotoxin levels were determined in particle supernatants by the limulus amebocyte lysate (LAL) assay as previously described [2, 7], and the $\mathrm{pH}$ of the particle supernatants was measured (Mettler Toledo AS, NY, USA).

2.3. Particle and Allergen Preparations. Ovalbumin (OVA, Gal d1; chicken egg albumin, grade VII, Sigma, St. Louis, MO, USA) was used as allergen after removal of endotoxin by Detoxi-Gel Endotoxin Removing Gel (Pierce, Rockford, IL, USA). The final endotoxin level measured less than $0.025 \mathrm{ng} / \mathrm{mg}$ OVA, as determined by the LAL assay. Particle suspensions were prepared in Hank's balanced salt solution (HBSS), with BALB/cA mouse serum to facilitate suspension of particles, and microtip probe sonicated as described in Nygaard et al. [2]. The CNT and CNF were even then mainly observed as agglomerates in TEM.

2.4. Injection Model. The IgE adjuvant capacity of the CNF particles was initially investigated in a footpad injection model suitable for studying respiratory allergy adjuvants (method reviewed in [13], performed as described in [2]). In short, groups of eight mice were given a single dose of $200 \mu \mathrm{g}$ of CNF A, B, C, D, or ufCBP together with $10 \mu \mathrm{g}$ OVA, OVA boosted $(10 \mu \mathrm{g})$ after 21 days and terminated on day 26 . Sera were collected and stored at $-20^{\circ} \mathrm{C}$ until quantification of OVA-specific IgE, IgG1, and IgG2a by ELISA (see below). A particle dose of $200 \mu \mathrm{g}$ was chosen, a dose previously shown to give a pronounced adjuvant effect of CNT in this injection model [2].

2.5. Intranasal Model. The adjuvant capacity of the particles after airway exposure was examined in an intranasal mouse model. Exposures were performed as described in [2], and a particle dose previously giving pronounced effects of CNT in the intranasal model was chosen [2]. In short, $133.3 \mu \mathrm{g}$ CNF A, B, C, D, swCNT, mwCNT, or ufCBP together with $10 \mu \mathrm{g}$ OVA was given intranasally to groups of ten mice on three consecutive days, giving a total dose of $400 \mu \mathrm{g}$ particles and $30 \mu \mathrm{g}$ OVA during sensitization. All mice were OVAboosted intranasally (10 $\mu$ g per day) on days 21,22 , and 23 . On day 26 , the animals were deeply anesthetized by a $0.3 \mathrm{~mL}$ intraperitoneal injection of a mixture of Zoletil forte $(17 \mathrm{mg} / \mathrm{kg}$ tiletamine and $17 \mathrm{mg} / \mathrm{kg}$ zolazepam; Virbac International, Carros Cedex, France) and Narcoxyl $(13.6 \mathrm{mg} / \mathrm{kg}$ xylazine; Intervet/Schering-Plough Animal Health, Boxmeer, The Netherlands). Sera were stored at $-20^{\circ} \mathrm{C}$ until quantification of OVA-specific IgE, IgG1, and IgG2a by ELISA (see below). Lung inflammation was determined by total and differential cell counts and cytokine levels in BALF (see below). Based on previous findings with sw and mw CNT [2], the cytokines TNF- $\alpha$ and MCP-1 in BALF were selected.

2.6. Detection of IgE, IgG1, and IgG2a Anti-OVA Antibodies. Detection of OVA-specific IgE, IgG1, and IgG2a antibodies was performed by ELISAs as described in Nygaard et al. [2]. The sera to be tested were diluted $1: 10$ for $\operatorname{IgE}$ and IgG2a, and for IgG1 diluted 1:4000 and 1:20000 for the 
injection and intranasal models, respectively. Values outside the dynamic range of the standard curve were set to a value just below/above the detection limit.

2.7. BALF Collection, Preparation, and Analyses. BALF was collected and prepared, and total and differential cell counts were determined as previously described $[2,14]$. In short, the supernatant from a first lavage was stored at $-80^{\circ} \mathrm{C}$ until cytokine measurements, while the cells from all three lavages were pooled, counted, and stained, and 400 cells per slide were differentiated into neutrophils, eosinophils, epithelial cells, lymphocytes, and macrophages. A number of macrophages appeared to contain particles, but since we could not determine whether the particles were taken up or were adsorbed to the cell membranes, they were counted as particle-associated macrophages. In BALF supernatants, the levels of tumor necrosis factor-alpha (TNF- $\alpha$ ) and monocyte chemoattractant protein-1 (MCP-1) were determined using BD Cytometric Bead Array (CBA) Mouse Soluble Protein Flex Sets, as previously described [2].

2.8. Data Analysis. The data were log10-transformed to meet the assumptions for running one-way ANOVAs. In order to determine which groups differed significantly from the others when the ANOVAs were positive $(P \leq 0.05)$, pairwise comparisons were performed by Tukey's post hoc test. Group differences were considered statistically significant if $P \leq$ 0.05. All statistical analyses were performed with SigmaStat Statistical Analysis System for Windows Version 2.03 (Jandel Scientific, Erkrath, Germany).

\section{Results}

3.1. Particle Characterization. Particle sample properties are shown in Table 1, while the methods used for particle characterization and more comprehensive characterization of the samples are previously reported [2, 7]. TEM micrographs at similar magnifications from CNF A, B, C, D, swCNT, and mwCNT samples are presented in Figures 1(a)-1(f), respectively. Systematic inspection of the TEM micrographs revealed that the majority of the $\mathrm{CNF}$ agglomerates were within the size range $0.6 \mu \mathrm{m}$ to $2.6 \mu \mathrm{m}$. The CNF were wider than the CNT, and the mwCNT were wider than the swCNT, as illustrated in the micrographs and given by the mean fiber width presented in Table 1. Most CNF are fractured or broken and therefore, on an average, are shorter than the CNT (as illustrated by dotted arrows in Figure 1, Table 1). Inspection of the TEM micrographs also showed that CNF samples and the swCNT sample contain disordered graphitic material, while the mwCNT sample is more homogeneous. High numbers of metal particles were observed in CNF A, CNF C, and swCNT, mainly embedded in the carbon material (indicated by arrows in Figures 1(c), 1(e), and 1(f)). CNF A and CNF C have a higher surface area, a lower mean fiber diameter, and a higher presence of structural defects than CNF B and CNF D samples (Table 1).

Figure 2 shows the typical internal structure of CNF and CNT in high-resolution TEM images. The CNT consist of single or multiple concentric graphene sheets rolled into form of cylinders (Figures 2(c) and 2(d)). In contrast, the CNF were classified into two main types of stacked arrangements of graphite cones: with channels that are mostly open (Figure 2(a)) or with periodically closed channels (Figure 2(b)). The latter are also characterized by closed layers on the CNF surface, as indicated by arrows. Although the open channel type was found in all CNF samples, only the CNF B and CNF D samples contained a high fraction of the periodically closed channel type. As previously reported [2], only half of the tubes in the swCNT sample were identified as single-walled, the other half being multiwalled. Most tubes in the mwCNT sample were defective, as exemplified in Figure 2(d) (arrowhead).

The particles were also studied after preparation in the medium used for the in vivo studies. As judged by TEM analyses, the sonication process did not affect the length of the CNT, whereas some breakage of the CNF was observed (data not shown). This was most probably due to a lower mechanical strength of the fibers compared to the tubes, caused by the different stacking of the graphite layers. The acidity $(\mathrm{pH})$ of the particle supernatants did not differ markedly between the particles, and the endotoxin levels were below detection limit for all particles, except swCNT which had a detectable but low level (Table 1).

3.2. OVA-Specific IgE, IgG1, and IgG2a Levels in Serum after Coinjection of Particles and OVA in the Footpad. In the footpad injection model, OVA-specific IgE levels were statistically significantly higher in mice treated with OVA and CNF A, CNF C, or ufCBP than with OVA alone (Figure 3(a)). ufCBP elicited significantly higher IgE levels than CNF B and $\mathrm{CNF}$ D, and CNF A elicited significantly higher levels than CNF D. OVA-specific IgG1 levels were significantly elevated by all particles, but CNF A and CNF C had higher IgG1-adjuvant activity than CNF B and D, but statistically significant only for CNF D (Figure 3(b)). CNF A, CNF C, and ufCBP gave weakly but significantly elevated levels of OVAspecific IgG2a levels (Figure 3(c)).

3.3. OVA-Specific IgE, IgG1, and IgG2a Levels in Serum after Intranasal Coexposure to Particles and OVA. In the intranasal model, all particles significantly increased OVA-specific IgE levels compared to the OVA control group (Figure 4(a)). The four CNF samples and ufCBP elicited similar levels of IgE, while in comparison, the sw and mw CNT elicited very high IgE levels. Also the OVA-specific IgG1 levels were significantly increased by all particles, and mwCNT elicited significantly higher levels than CNF A and CNF B (Figure 4(b)). As compared to the OVA control group, OVA-specific IgG2a levels were significantly increased after exposure to CNF $\mathrm{C}$ and mwCNT and also tended to be increased by swCNT $(P=0.079)$ and ufCBP exposure $(P=$ 0.088; Figure 4(c)).

\subsection{Airway Inflammation after Intranasal Coexposure to} Particles and OVA. In the intranasally exposed mice, CNF B, CNF C, sw and mw CNT, and ufCBP with OVA significantly increased the total number of BALF cells, compared to the 


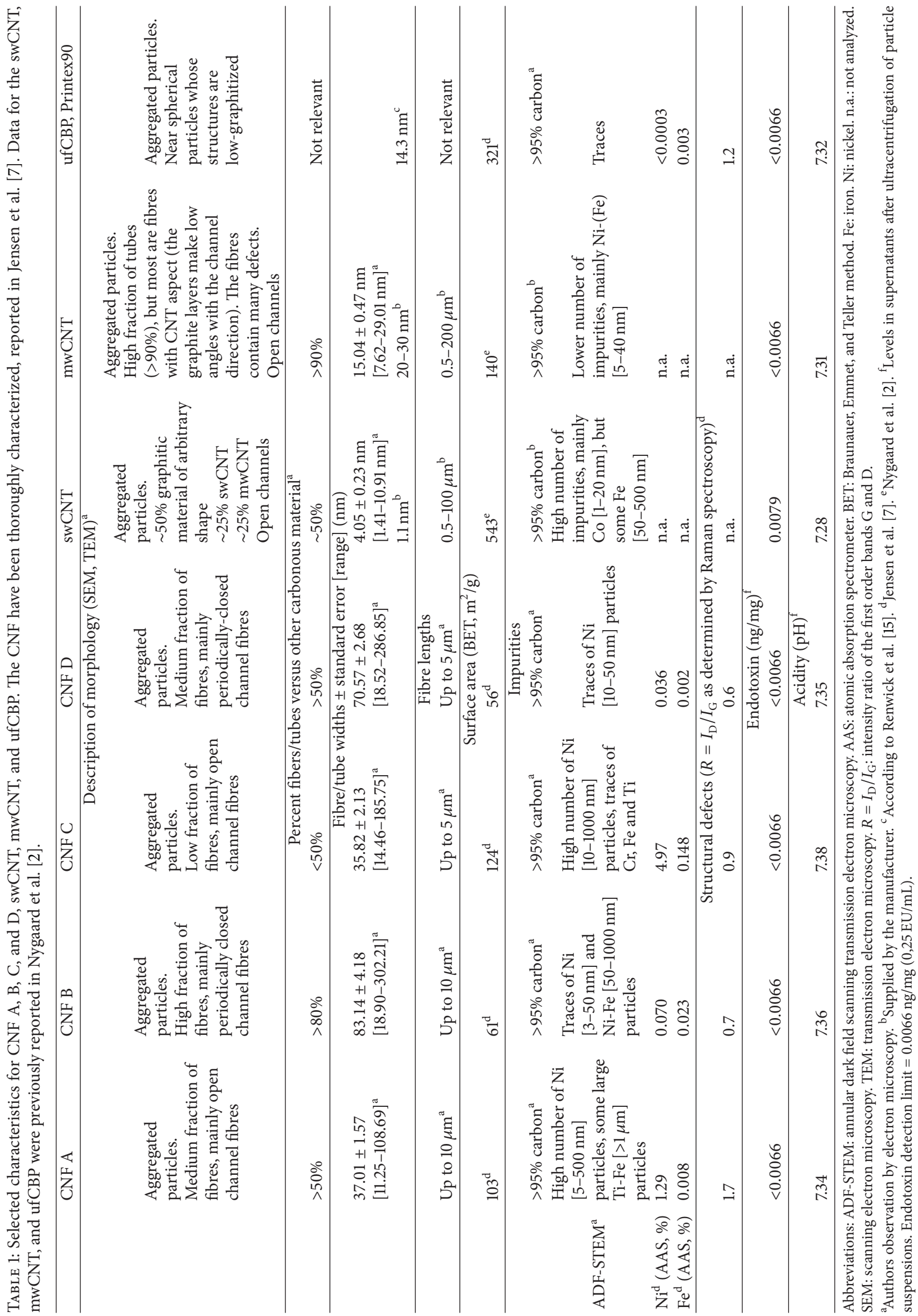




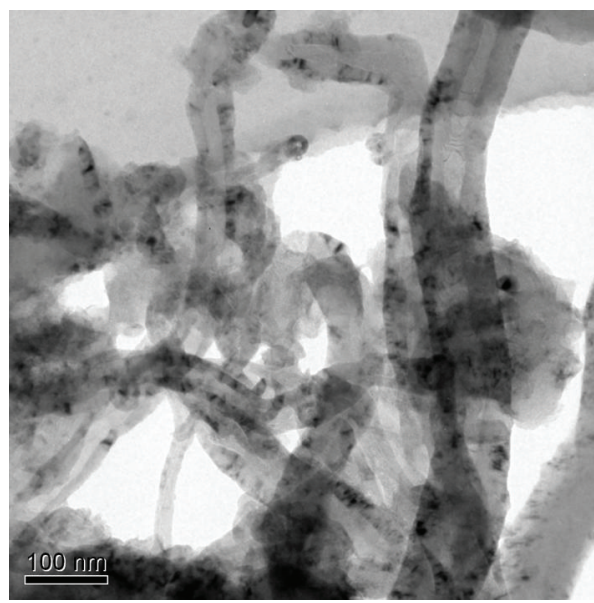

(a)

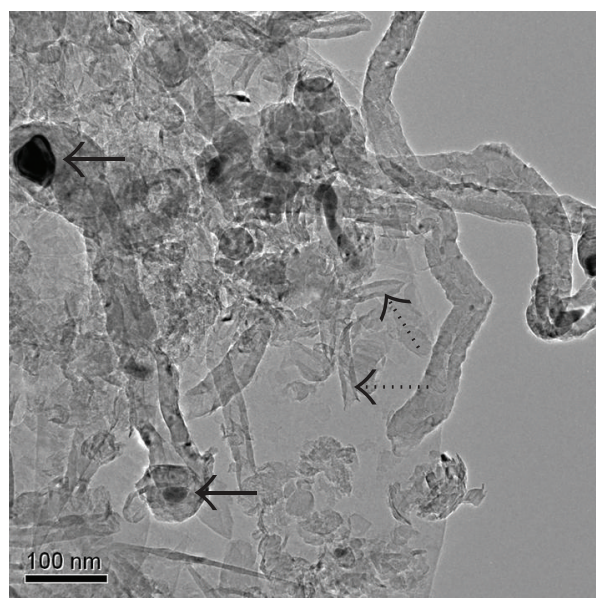

(c)

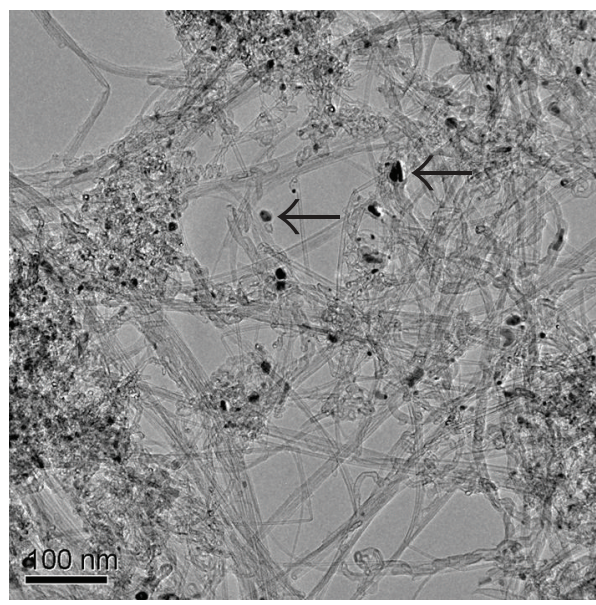

(e)

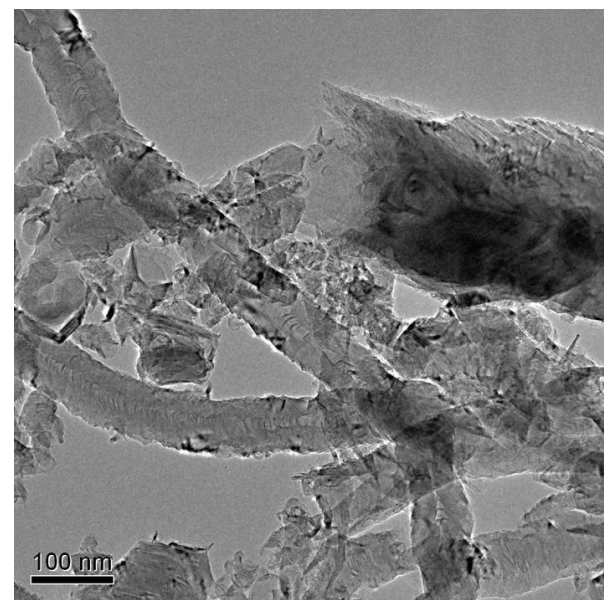

(b)

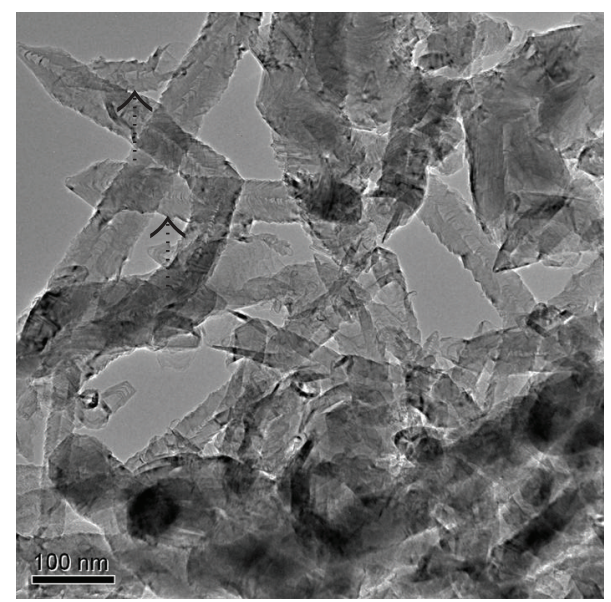

(d)

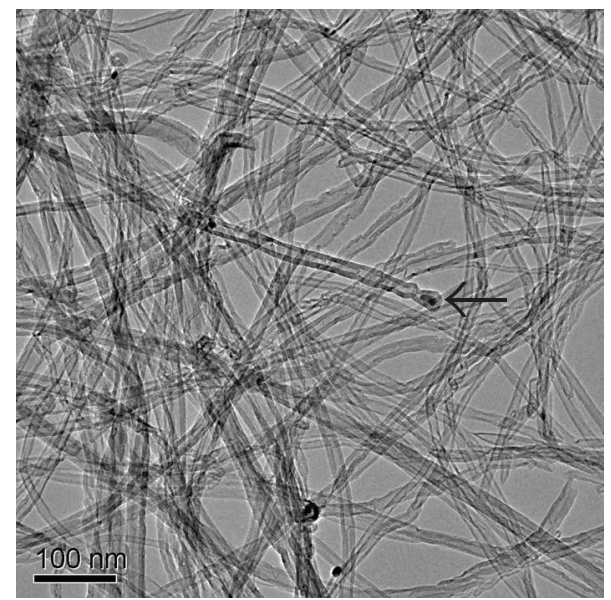

(f)

FIGURE 1: TEM micrographs of the particle samples. Micrographs of CNF A (a), CNF B (b), CNF C (c), CNF D (d), swCNT (e), and mwCNT (f) illustrate the difference in width and length between the CNF and CNT. Examples of metallic particle presence are indicated by dense arrows. Most metallic particles are embedded in the carbon material. Shorter fragments of fibers are indicated by dotted arrows. 


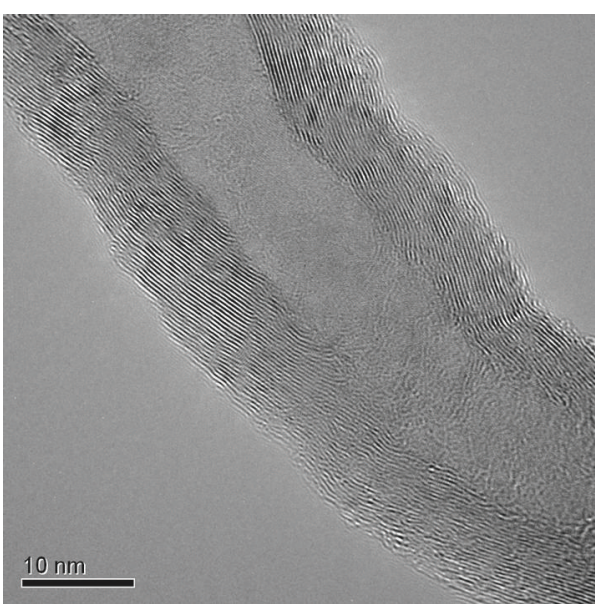

(a) Open-channel CNF

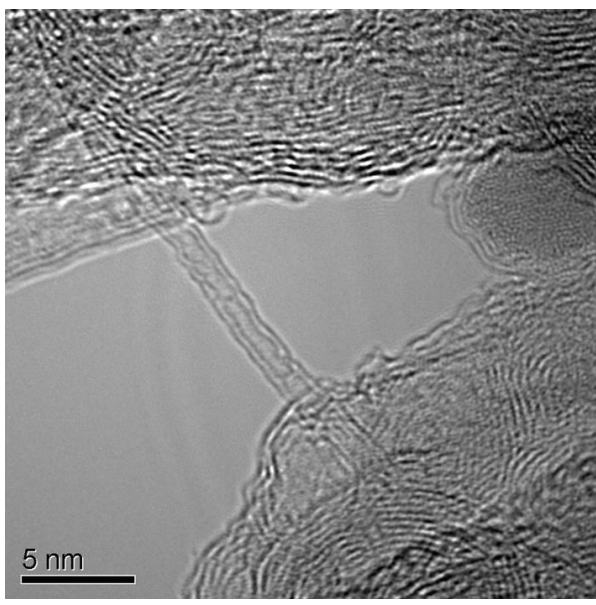

(c) swCNT

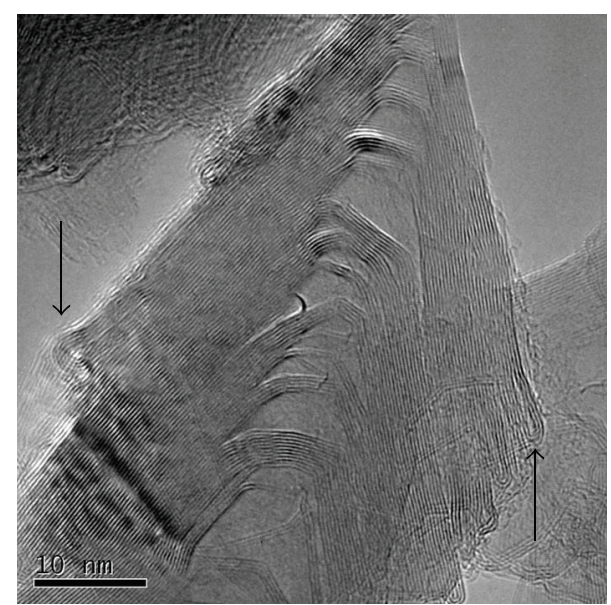

(b) Periodically closed CNF

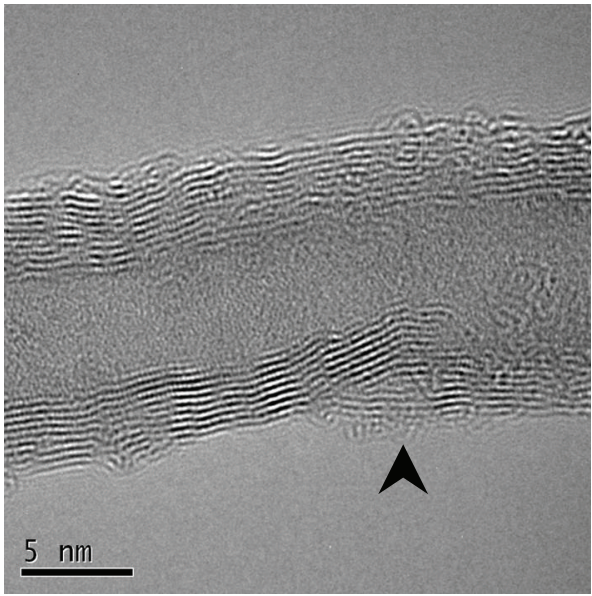

(d) $\mathrm{mwCNT}$

FIGURE 2: High-resolution images of particles, illustrating their internal structure. The herringbone CNFs consisted of graphite layers arranged at an angle to the axis of the filament structure ((a), (b)). The micrographs show the structure of a typical open-channel fiber found in all CNF samples (a) and a periodically closed fiber present in large amounts only in CNF B and CNF D (b). Many graphite layers in the periodicallyclosed type of fiber have closed ends at the surface, indicated by arrows in (b). A typical example of a swCNT, together with disordered graphitic material, is shown in (c). An example of a typical defect mwCNT is shown in (d); a defect in the wall is indicated by an arrow head.

group given OVA alone (Figure 5(a)). The total cell numbers were significantly higher in the groups given swCNT, mwCNT, and ufCBP than in the CNF groups, with the exception of CNF C which elicited significantly higher cell numbers than CNF A and CNF D. The particles that increased the total cell numbers also significantly increased the neutrophil cell numbers (Figure 5(b)). The eosinophil numbers were low in the groups treated with OVA alone or together with the CNF samples. Exposure to OVA with swCNT or mwCNT, however, induced significantly higher eosinophil numbers than OVA alone or OVA with any of the CNF samples (Figure 5(c)). ufCBP with OVA also increased the eosinophil numbers, although the numbers were only significantly higher than in mice treated with OVA alone or together with CNF $A$ and CNF B. The number of lymphocytes was slightly, but statistically significantly, increased in the swCNT group only (Figure 5(d)), and the epithelial cell numbers were only marginally affected (data not shown). swCNT, mwCNT, and
ufCBP with OVA significantly increased the macrophage numbers compared to OVA alone (Figure 5(e)), while CNF $\mathrm{C}$ was the only CNF sample that significantly increased the macrophage numbers. The percentage of macrophages associated with particles did not differ markedly between the different particle types (Figure 5(f)). However, the CNF D, swCNT, mwCNT, and ufCBP tended to be associated with a lower percentage of macrophages than $\mathrm{CNF} A, \mathrm{~B}$, and $\mathrm{C}$ and were significantly different for swCNT and ufCBP only, as compared to CNF B.

Due to accidental thawing, only half of the BALF samples were analyzed for cytokines, reducing the ability to detect statistically significant differences. The levels of MCP-1 after OVA booster apparently were increased by all particles with OVA except CNF B (Figure 5(g)). The tendencies were most pronounced for CNF A, CNF C, mwCNT, and ufCBP, although statistical significance was reached only for the latter. Although the levels of TNF- $\alpha$ were low at this time 


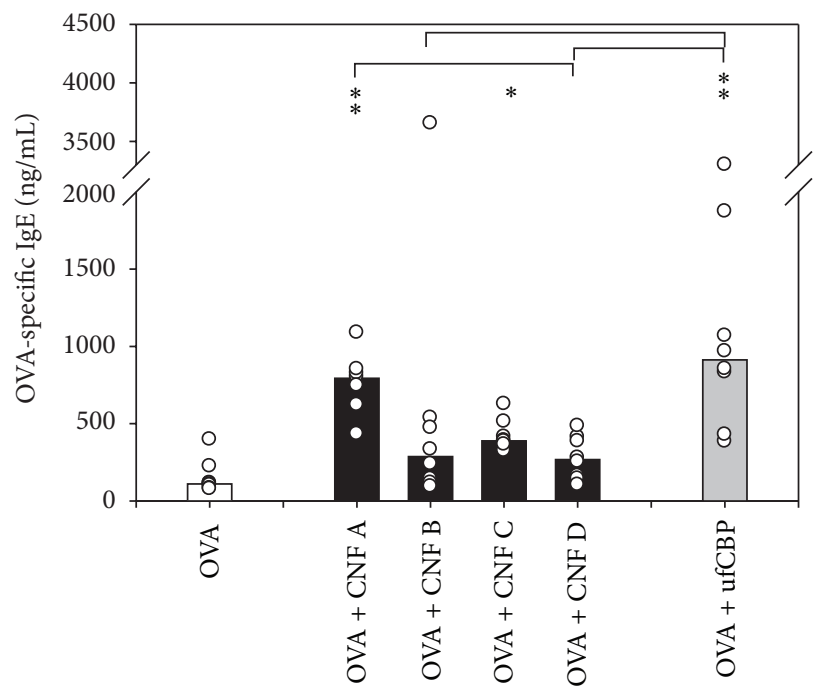

(a) $\operatorname{IgE}$

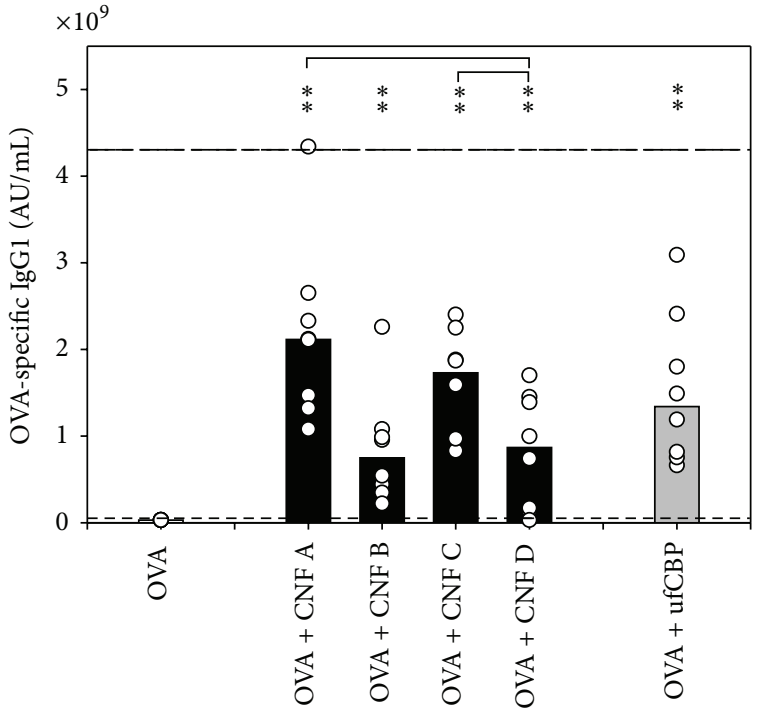

(b) $\operatorname{IgG1}$

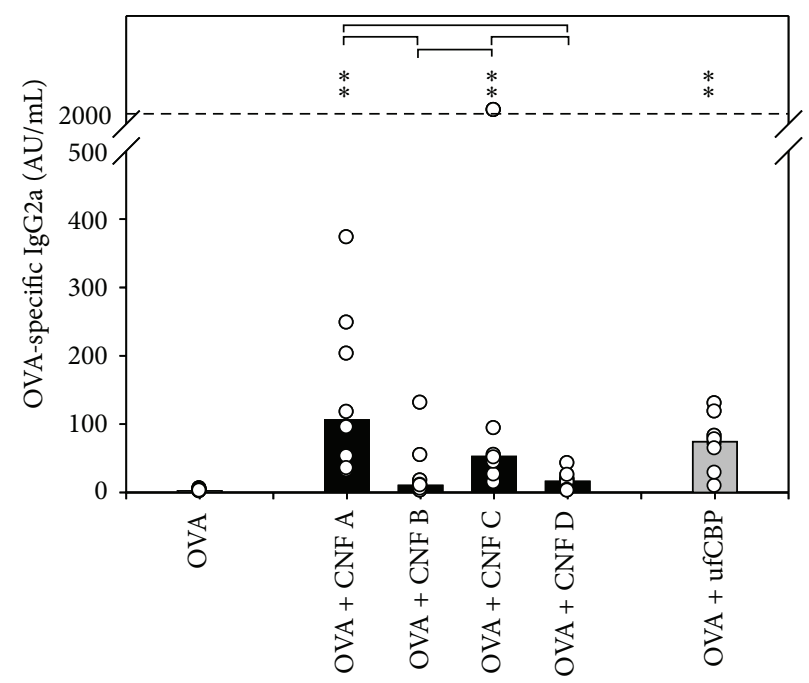

(c) IgG2a

FIgURE 3: Allergen-specific antibodies after subcutaneous coinjection of allergen and particles. Serum levels of OVA-specific IgE (a), IgG1 (b), and IgG2a (c) 26 days after subcutaneous injection into one hind footpad of $10 \mu \mathrm{g}$ OVA alone (white bars) or together with $200 \mu \mathrm{g}$ CNF (samples A, B, C, and D; black bars) or ufCBP (light grey bars). On day 21, all mice were boosted with $10 \mu \mathrm{g}$ OVA in the footpad. Values (ng or arbitrary units (AU) per $\mathrm{mL}$ ) for individual mice (circles) and median values (columns) for groups of eight mice are shown. If values were outside the dynamic range of the ELISA assays, the dotted lines indicate the lower or upper quantitative detection limits for the assays. Asterisk denotes significant differences compared to the OVA group, ${ }^{* *} P<0.001,{ }^{*} P<0.05$. Brackets denote statistically significant differences between the particle groups, $P<0.05$.

point (23 days after particle exposure), CNF A and ufCBP with OVA tended to induce higher levels than the other groups (Figure $5(\mathrm{~h})$ ).

\section{Discussion}

Studies of airway effects of CNF are scarce in the literature. In the present study, we demonstrate that CNF can promote production of allergen-specific IgE after injection and intranasal exposure to mice. While CNF A and CNF C, and not CNF $\mathrm{B}$ and CNF D, demonstrated IgE adjuvant capacity in the injection model, all four CNF samples elicited similar levels of $\operatorname{IgE}$ in the airway model. The increase in IgE levels was not associated with a clear allergic airway inflammation in the intranasal model used, since no significant eosinophil influx was observed after allergen booster. Our observation that CNF promote antibody production is in agreement with previous studies reporting allergy-promoting activity of nanosized particles like ultrafine polystyrene particles, ufCBP and CNT $[1,2,10,11,16]$.

In the intranasal model, the sw and mw CNT samples elicited markedly higher IgE levels than the CNF samples, accompanied with a clear eosinophil airway inflammation. 


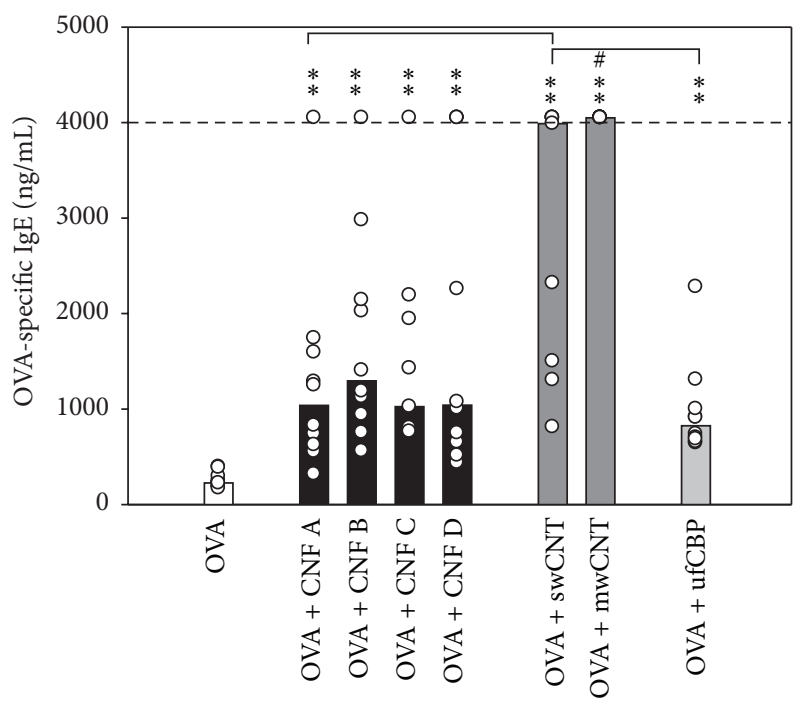

(a) $\operatorname{IgE}$

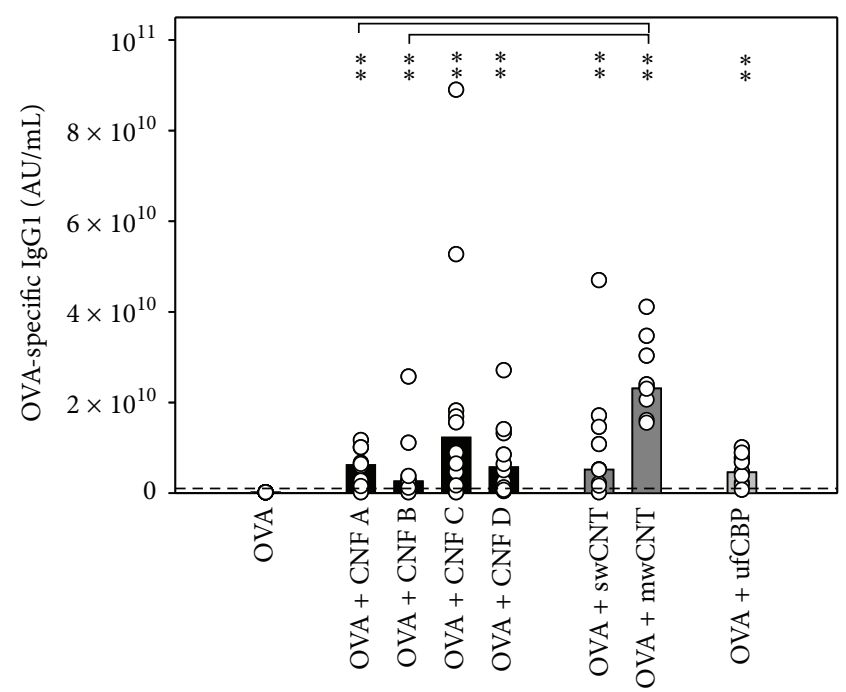

(b) $\operatorname{IgG1}$

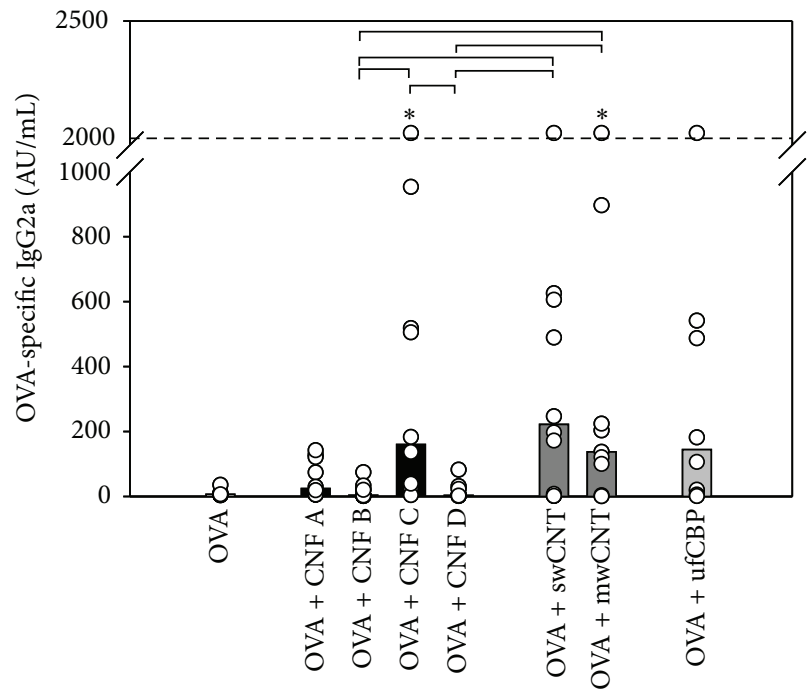

(c) IgG2a

Figure 4: Allergen-specific antibodies after intranasal coexposure to allergen and particles. Serum levels of OVA-specific IgE (a), IgG1 (b), and IgG2a (c) 26 days after intranasal exposure to $10 \mu \mathrm{g}$ OVA alone (white bars) or together with $133 \mu \mathrm{g}$ particles on days 0,1 and 2 (total dose of $30 \mu \mathrm{g}$ OVA and $400 \mu \mathrm{g}$ particles). The four samples of CNF (black bars), swCNT and mwCNT (dark grey bars), and ufCBP (light grey bars) were used. On days 21,22 , and 23 , all mice were boosted intranasally with $10 \mu \mathrm{g}$ OVA (per day). Values (ng or arbitrary units (AU) per mL) for individual mice (circles) and median values (columns) for groups of ten mice are shown. If values were outside the dynamic range of the ELISA assays, the dotted lines indicate the lower or upper quantitative detection limits for the assays. Asterisk denotes significant differences compared to the OVA group, ${ }^{* *} P<0.001,{ }^{*} P<0.05$. \# Denotes significant differences compared to all other particle groups, $P<0.05$. Brackets denote statistically significant differences between particle groups, $P<0.05$.

The features of the two CNT samples, making them different from the CNF samples, are the presence of long, thin, and hollow structures (tubes), as opposed to the wider, hat-stacked, more compact structures of the CNF (Figure 1, Table 2). In addition, the present CNT are longer and have $1 / 2-1 / 20$ of the diameter as compared to the CNF, giving them a higher aspect ratio (i.e., the length/width ratio). Particles with high aspect ratios, such as asbestos fibers, have demonstrated high toxicity, and CNT have been reported to have pathogenic features similar to asbestos fibers [17]. The main characteristic distinguishing nanofibers from nanotubes is the stacking of graphene sheets of varying shapes [18]. While sw and mw CNT consist of single or multiple graphene sheets rolled into concentric cylinders, herringbone (hat-stacked) CNF consist of graphite layers arranged at an angle to the axis of the filament structure, forming a stacked arrangement of cones (Figure 2). This intrinsic difference results in less mechanical strength of CNF compared to CNT [18], as also observed in our study, since the CNF were more susceptible to breaking during the sonication process. In contrast to what has been 


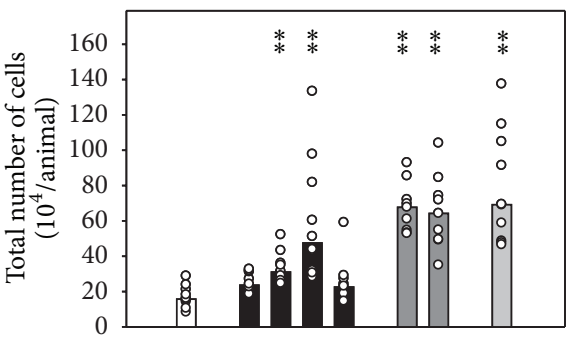

(a) Total cell numbers

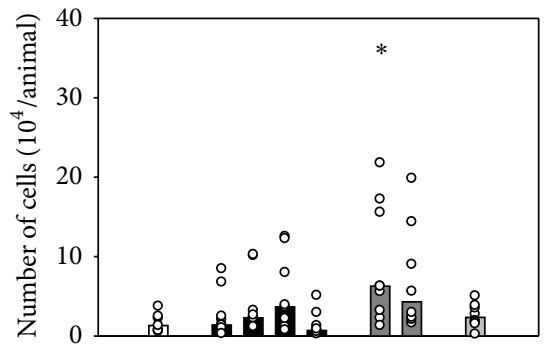

(d) Lymphocytes

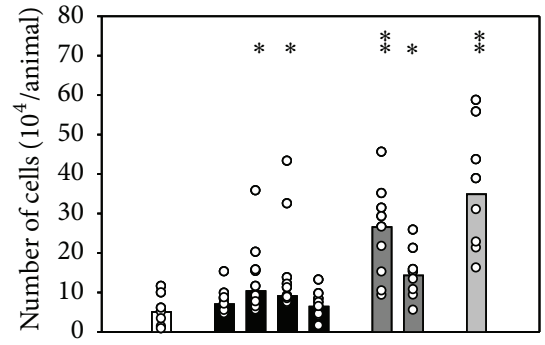

(b) Neutrophils

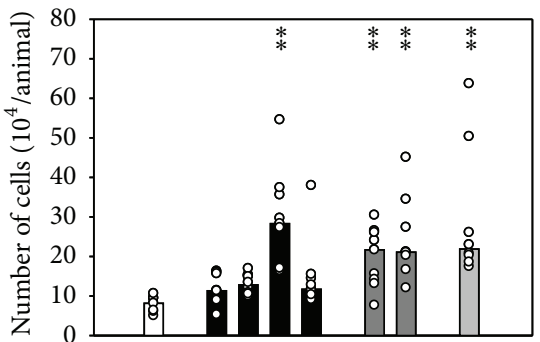

(e) Macrophages

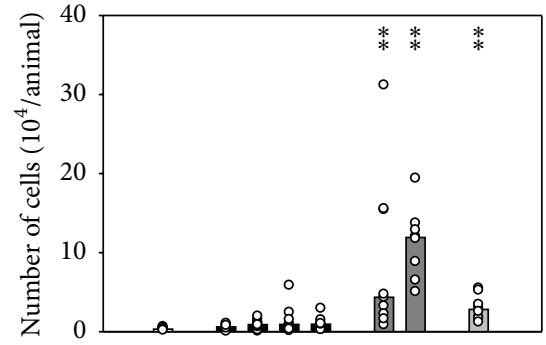

(c) Eosinophils

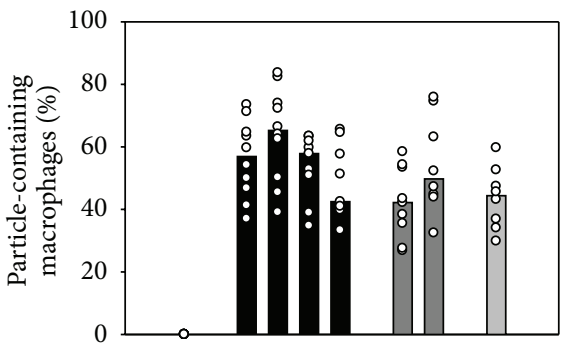

(f) Particle-associated macrophages

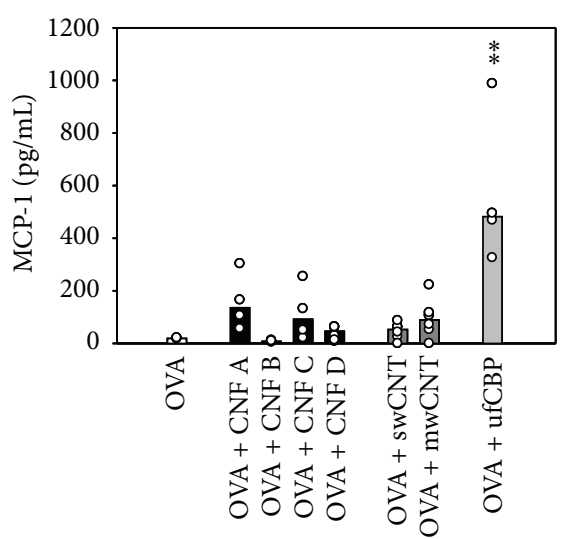

(g) MCP-1

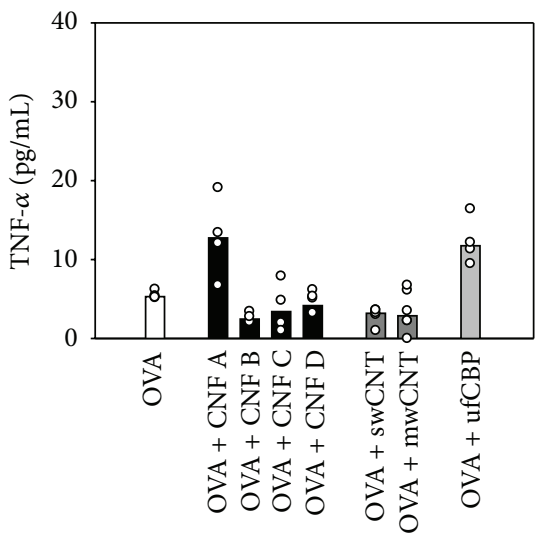

(h) TNF- $\alpha$

FIGURE 5: Airway inflammation measured after intranasal coexposure to allergen and particles. Cell numbers and cytokines in BALF collected following OVA booster, 26 days after intranasal exposure of mice to allergen alone or together with particles (as described in legend of Figure 4). The total cell numbers (a) were determined on a Coulter cell counter, whereas the number of neutrophils (b), eosinophils (c), lymphocytes (d), and macrophages (e) and the percent of particle-associated macrophages (f) were determined by counting 400 cells per stained cytoslide. The amount of MCP-1 (g) and TNF- $\gamma(\mathrm{h})$ in BALF supernatants was determined by CBA assay. Values for individual mice (circles) and group medians (columns) for groups of ten mice are shown, except MCP-1 and TNF- $\alpha$ where only half of the samples could be measured. Asterisk denotes significant differences compared to the OVA group, ${ }^{* *} P<0.001,{ }^{*} P<0.05$. Statistically significant differences between particle groups are given in the results section.

observed for mwCNT [19], hat-stacked CNF implanted in subcutaneous tissue of rats appeared to become shorter and display decreased degree of aggregation over time [20]. The authors suggested that delamination of graphene layers by hydrophilic substances or energy from cytoplasmic motion may be involved in CNF shortening. Thus, although studies have demonstrated some biodegradation in vitro [21], the present and previous data suggest that the CNF samples have lower biopersistence than the CNT samples.

Further, the open, hollow structure of the CNT may be of importance for the allergy-promoting capacity, for instance, as a depot of allergens. Carbon particles have previously been shown to act as allergen carriers [22, 23], and a depot effect has been suggested as one mechanism for particle adjuvant effects [24]. The above mentioned reports support the present finding that tube-associated characteristics, such as a thin, hollow tube structure with assumed high biopersistence, appeared to be particularly important features for the allergy-promoting effect of particles in the airways. CNT also generate reactive oxygen species [25], which probably plays an additional role in their induction of airway allergic inflammation. The capacity of CNF to form ROS is unknown.

Knowledge on which particle characteristics are important for the different biological effects is a prerequisite to enable production of less toxic particles [26]. An advantage of this study was the availability of four CNF batches 
TABLE 2: Distribution of selected particle characteristics and the allergy-promoting capacity for all particles tested (as measured by high levels of OVA-specific IgE and eosinophil airway inflammation). To simplify the identification of particle characteristics of importance for the modulated allergy responses after airway exposure, the levels of each particle property or allergic response are subjectively categorized into three levels, illustrated by roman, bold italic, or bold font.

\begin{tabular}{|c|c|c|c|c|c|c|c|}
\hline & $\begin{array}{l}\text { Main carbon } \\
\text { structure }\end{array}$ & $\begin{array}{c}\text { Fraction of } \\
\text { fibers/tubes versus } \\
\text { disordered material }\end{array}$ & $\begin{array}{l}\text { Fiber/tube } \\
\text { width }(\mathrm{nm})\end{array}$ & $\begin{array}{c}\text { Surface area } \\
\left(\mathrm{m}^{2} / \mathrm{g}\right)\end{array}$ & $\begin{array}{c}\text { Structural } \\
\text { defects } \\
\left(R=I_{\mathrm{D}} / I_{\mathrm{G}}\right)\end{array}$ & $\begin{array}{c}\text { Metallic } \\
\text { contaminants }\end{array}$ & $\begin{array}{l}\text { Allergy-promoting } \\
\text { capacity }\end{array}$ \\
\hline CNF A & $\begin{array}{l}\text { Fibers, open } \\
\text { channels }\end{array}$ & Medium & $\begin{array}{c}37.01 \pm 1.57 \\
{[11.25-108.69]}\end{array}$ & 103 & 1.7 & $\begin{array}{c}\text { High, mainly } \\
\mathrm{Ni}\end{array}$ & Medium \\
\hline CNF B & $\begin{array}{c}\text { Fibers, } \\
\text { periodically } \\
\text { closed channels }\end{array}$ & High & $\begin{array}{c}83.14 \pm 4.18 \\
{[18.90-302.21]}\end{array}$ & 61 & 0.7 & Traces & Medium \\
\hline CNF C & $\begin{array}{c}\text { Fibers, open } \\
\text { channels }\end{array}$ & Low & $\begin{array}{c}35.82 \pm 2.13 \\
{[14.46-185.75]}\end{array}$ & 124 & 0.9 & $\begin{array}{c}\text { High, mainly } \\
\text { Ni }\end{array}$ & Medium \\
\hline CNF D & $\begin{array}{l}\text { Fibers, periodically } \\
\text { closed channels }\end{array}$ & Medium & $\begin{array}{c}70.57 \pm 2.68 \\
{[18.52-286.85]}\end{array}$ & 56 & 0.6 & Traces & Medium \\
\hline swCNT & $\begin{array}{l}\text { Tubes, open } \\
\text { channels }\end{array}$ & Medium & $\begin{array}{c}4.05 \pm 0.23 \\
{[1.41-10.91 \mathrm{~nm}]}\end{array}$ & 543 & n.a. & $\begin{array}{l}\text { High, mainly } \\
\text { Co }\end{array}$ & High \\
\hline mwCNT & $\begin{array}{c}\text { Tubes, open } \\
\text { channels }\end{array}$ & High & $\begin{array}{c}15.04 \pm 0.47 \\
{[7.62-29.01 \mathrm{~nm}]}\end{array}$ & 140 & n.a. & $\begin{array}{l}\text { Less, mainly } \\
\mathrm{Ni}(\mathrm{Fe})\end{array}$ & High \\
\hline ufCBP & Spherical & Not relevant & Not relevant & 321 & 1.2 & Traces & Medium \\
\hline
\end{tabular}

manufactured in the same facility but with varying particle characteristics deliberately introduced by changing manufacturing conditions [7]. Despite their qualitative differences, the allergy-promoting capacity of the four CNF samples did not differ in the intranasal model, neither with regard to $\operatorname{IgE}$ levels nor eosinophil influx. In the footpad injection model, on the other hand, only the CNF A and CNF C increased OVA-specific IgE levels. CNF $\mathrm{A}$ and $\mathrm{CNF} \mathrm{C}$ also tended to differ from the other CNF samples with regard to other endpoints, such as higher levels of IgG1 and IgG2a antibodies in both models and some of the inflammatory markers in the BALF. Although the biological implications of these observations are unclear, they indicate that the CNF A and CNF C samples differ from the CNF B and CNF D samples with regard to induction of biological responses. Common for CNF A and CNF C is a higher metal content, about twice as large relative surface area, a lower mean fiber width, a higher proportion of open channel fibers, and a tendency towards a higher number of defective sites compared to CNF $\mathrm{B}$ and CNF D (Table 2). These are all parameters affected by the high temperature treatment performed to reduce the metal content in CNF B and CNF D (described in [7]), and our results therefore suggest that heat-treated CNF may be less toxic with regard to some biological responses. Unfortunately, the association between these heat-treatment sensitive parameters makes it hard to identify single key properties responsible for the higher responses to the CNF A and CNF C. Both metal content, surface area, and structural defects have been suggested to play a role in particle-induced lung inflammation and allergy-promoting effects in mice [16, 27-32]. However, we cannot exclude that the lower fiber mean width and the higher proportion of open channeled fibers in the CNF A and CNF C samples may have contributed to the stronger responses to these particles. Indeed, this would be in agreement with the apparent importance of a thin, hollow structure on the allergic adjuvant effect, as discussed previously.

\section{Conclusion}

The present data demonstrate that $\mathrm{CNF}$ and CNT modulate airway responses to allergens, resulting in allergic airway inflammation and production of allergen-specific IgE in mice. When different CNF samples were compared with swCNT and mwCNT, however, the CNT samples appeared to be especially potent in promoting allergic responses, possibly due to their thin, hollow tube structure and assumed high biopersistence. This study provides a basis for studies aiming to further identify the particle properties of importance for airway effects and studies aiming to better understand which airway mechanisms are underlying the allergy-promoting effect of nanoparticles such as CNF and CNT.

\section{Acknowledgments}

The authors gratefully acknowledge Else-Carin Groeng and Trude Karin Olsen for excellent technical assistance, as well as the help from Åse Eikeset, Bodil Hasseltvedt and Berit A. Stensby, and the staff of the animal facilities. Many interesting interdisciplinary discussions with Sten Yngve Larsen, Ellen Katrine Jensen, and Tore Syversen are highly acknowledged, as well as the generous provision of CNF from Statoil and Elkem Carbon AS and Printex90 particles by Degussa. The authors also thank Ellen Namork for critical reading of the manuscript. This work was partially financed by the project "Carbon fiber and health" with joint funding from Statoil ASA, Elkem Carbon AS, The Research Council of Norway (Grant no. 176425/S10), and the Norwegian Institute of Public Health. The funding bodies did not have control over the 
present study design, interpretation of data, or the resulting publication.

\section{References}

[1] C. De Haar, I. Hassing, M. Bol, R. Bleumink, and R. Pieters, "Ultrafine but not fine particulate matter causes airway inflammation and allergic airway sensitization to co-administered antigen in mice," Clinical and Experimental Allergy, vol. 36, no. 11, pp. 1469-1479, 2006.

[2] U. C. Nygaard, J. S. Hansen, M. Samuelsen, T. Alberg, C. D. Marioara, and M. Løvik, "Single-walled and multi-walled carbon nanotubes promote allergic immune responses in mice," Toxicological Sciences, vol. 109, no. 1, pp. 113-123, 2009.

[3] B. Granum and M. Løvik, "The effect of particles on allergic immune responses," Toxicological Sciences, vol. 65, no. 1, pp. 717, 2002.

[4] D. Diaz-Sanchez, "The role of diesel exhaust particles and their associated polyaromatic hydrocarbons in the induction of allergic airway disease," Allergy, vol. 52, no. 38, pp. 52-56, 1997.

[5] S. F. Hansen, E. S. Michelson, A. Kamper, P. Borling, F. StuerLauridsen, and A. Baun, "Categorization framework to aid exposure assessment of nanomaterials in consumer products," Ecotoxicology, vol. 17, no. 5, pp. 438-447, 2008.

[6] J. J. Bang, P. A. Guerrero, D. A. Lopez, L. E. Murr, and E. V. Esquivel, "Carbon nanotubes and other fullerene nanocrystals in domestic propane and natural gas combustion streams," Journal of Nanoscience and Nanotechnology, vol. 4, no. 7, pp. 716-718, 2004.

[7] E. K. Jensen, S. Y. Larsen, U. C. Nygaard, C. D. Marioara, and T. Syversen, "Early combination of material characteristics and toxicology is useful in the design of low toxicity carbon nanofiber," Materials, vol. 5, pp. 1560-1580, 2012.

[8] A. A. Shvedova and V. E. Kagan, "The role of nanotoxicology in realizing the 'helping without harm' paradigm of nanomedicine: lessons from studies of pulmonary effects of single-walled carbon nanotubes," Journal of Internal Medicine, vol. 267, no. 1, pp. 106-118, 2010.

[9] J. P. Ryman-Rasmussen, E. W. Tewksbury, O. R. Moss, M. F. Cesta, B. A. Wong, and J. C. Bonner, "Inhaled multiwalled carbon nanotubes potentiate airway fibrosis in murine allergic asthma," American Journal of Respiratory Cell and Molecular Biology, vol. 40, no. 3, pp. 349-358, 2009.

[10] K.-I. Inoue, E. Koike, R. Yanagisawa, S. Hirano, M. Nishikawa, and H. Takano, "Effects of multi-walled carbon nanotubes on a murine allergic airway inflammation model," Toxicology and Applied Pharmacology, vol. 237, no. 3, pp. 306-316, 2009.

[11] K.-I. Inoue, R. Yanagisawa, E. Koike, M. Nishikawa, and H. Takano, "Repeated pulmonary exposure to single-walled carbon nanotubes exacerbates allergic inflammation of the airway: possible role of oxidative stress," Free Radical Biology and Medicine, vol. 48, no. 7, pp. 924-934, 2010.

[12] E.-J. Park, W.-S. Cho, J. Jeong, J. Yi, K. Choi, and K. Park, "Proinflammatory and potential allergic responses resulting from $\mathrm{B}$ cell activation in mice treated with multi-walled carbon nanotubes by intratracheal instillation," Toxicology, vol. 259, no. 3, pp. 113-121, 2009.

[13] M. Løvik, T. Alberg, U. C. Nygaard, M. Samuelsen, E.-C. Groeng, and P. I. Gaarder, "Popliteal lymph node (PLN) assay to study adjuvant effects on respiratory allergy," Methods, vol. 41, no. 1, pp. 72-79, 2007.
[14] M. Samuelsen, U. C. Nygaard, and M. Løvik, "Particle size determines activation of the innate immune system in the Lung," Scandinavian Journal of Immunology, vol. 69, no. 5, pp. 421-428, 2009.

[15] L. C. Renwick, D. Brown, A. Clouter, and K. Donaldson, "Increased inflammation and altered macrophage chemotactic responses caused by two ultrafine particle types," Occupational and Environmental Medicine, vol. 61, pp. 442-447, 2004.

[16] U. C. Nygaar, M. Samuelsen, A. Aase, and M. Løvik, “The capacity of particles to increase allergic sensitization is predicted by particle number and surface area, not by particle mass," Toxicological Sciences, vol. 82, no. 2, pp. 515-524, 2004.

[17] C. A. Poland, R. Duffin, I. Kinloch et al., "Carbon nanotubes introduced into the abdominal cavity of mice show asbestoslike pathogenicity in a pilot study," Nature Nanotechnology, vol. 3, no. 7, pp. 423-428, 2008.

[18] A. V. Melechko, V. I. Merkulov, T. E. McKnight et al., "Vertically aligned carbon nanofibers and related structures: controlled synthesis and directed assembly," Journal of Applied Physics, vol. 97, no. 4, Article ID 041301, 2005.

[19] J. Pauluhn, "Subchronic 13-week inhalation exposure of rats to multiwalled carbon nanotubes: toxic effects are determined by density of agglomerate structures, not fibrillar structures," Toxicological Sciences, vol. 113, no. 1, pp. 226-242, 2010.

[20] A. Yokoyama, Y. Sato, Y. Nodasaka et al., "Biological behavior of hat-stacked carbon nanofibers in the subcutaneous tissue in rats," Nano Letters, vol. 5, no. 1, pp. 157-161, 2005.

[21] V. E. Kagan, N. V. Konduru, W. Feng et al., "Carbon nanotubes degraded by neutrophil myeloperoxidase induce less pulmonary inflammation," Nature Nanotechnology, vol. 5, no. 5, pp. 354-359, 2010.

[22] H. Ormstad, B. V. Johansen, and P. I. Gaarder, "Airborne house dust particles and diesel exhaust particles as allergen carriers," Clinical and Experimental Allergy, vol. 28, no. 6, pp. 702-708, 1998.

[23] R. B. Knox, C. Suphioglu, P. Taylor et al., "Major grass pollen allergen Lol $\mathrm{p} 1$ binds to diesel axhaust particles: implications for asthma and air pollution," Clinical and Experimental Allergy, vol. 27, no. 3, pp. 246-251, 1997.

[24] S. Parnia, J. L. Brown, and A. J. Frew, "The role of pollutants in allergic sensitization and the development of asthma," Allergy, vol. 57, no. 12, pp. 1111-1117, 2002.

[25] A. A. Shvedova, E. Kisin, A. R. Murray et al., "Inhalation vs. aspiration of single-walled carbon nanotubes in C57BL/6 mice: inflammation, fibrosis, oxidative stress, and mutagenesis," American Journal of Physiology, vol. 295, no. 4, pp. L552-L565, 2008.

[26] R. H. Hurt, M. Monthioux, and A. Kane, "Toxicology of carbon nanomaterials: status, trends, and perspectives on the special issue," Carbon, vol. 44, no. 6, pp. 1028-1033, 2006.

[27] D. M. Brown, M. R. Wilson, W. MacNee, V. Stone, and K. Donaldson, "Size-dependent proinflammatory effects of ultrafine polystyrene particles: a role for surface area and oxidative stress in the enhanced activity of ultrafines," Toxicology and Applied Pharmacology, vol. 175, no. 3, pp. 191-199, 2001.

[28] I. Fenoglio, G. Greco, M. Tomatis et al., "Structural defects play a major role in the acute lung toxicity of multiwall carbon nanotubes: physicochemical aspects," Chemical Research in Toxicology, vol. 21, no. 9, pp. 1690-1697, 2008.

[29] S. H. Gavett, N. Haykal-Coates, L. B. Copeland, J. Heinrich, and M. I. Gilmour, "Metal composition of ambient PM2.5 influences 
severity of allergic airways disease in mice," Environmental Health Perspectives, vol. 111, no. 12, pp. 1471-1477, 2003.

[30] B. Granum, P. I. Gaarder, and M. Lovik, "IgE adjuvant activity of particles-what physical characteristics are important?" Inhalation Toxicology, vol. 12, no. 3, pp. 365-372, 2000.

[31] A. L. Lambert, W. Dong, M. K. Selgrade, and M. I. Gilmour, "Enhanced allergic sensitization by residual oil fly ash particles is mediated by soluble metal constituents," Toxicology and Applied Pharmacology, vol. 165, no. 1, pp. 84-93, 2000.

[32] T. Stoeger, C. Reinhard, S. Takenaka et al., "Instillation of six different ultrafine carbon particles indicates a surface area threshold dose for acute lung inflammation in mice," Environmental Health Perspectives, vol. 114, no. 3, pp. 328-333, 2006. 


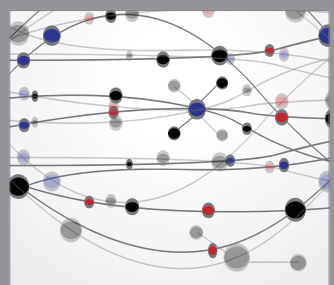

The Scientific World Journal
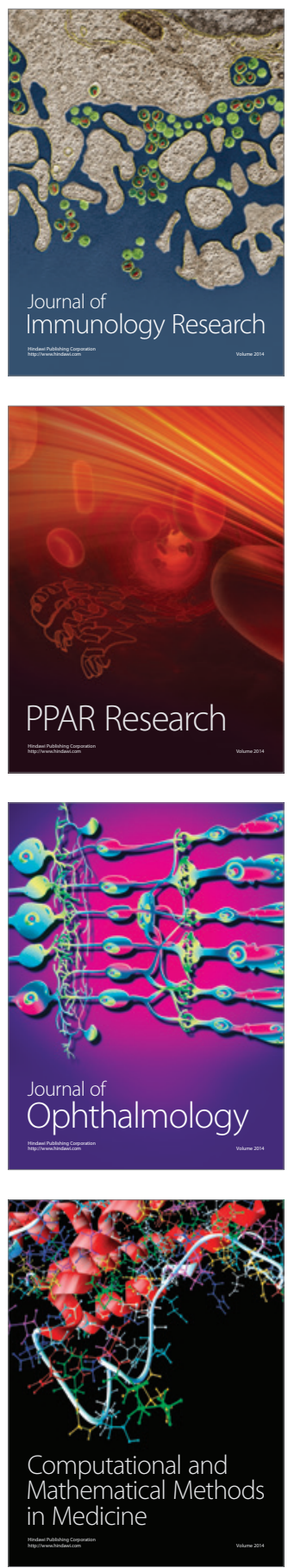

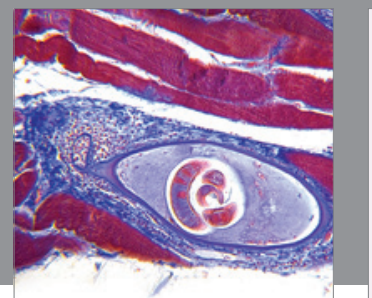

Gastroenterology

Research and Practice
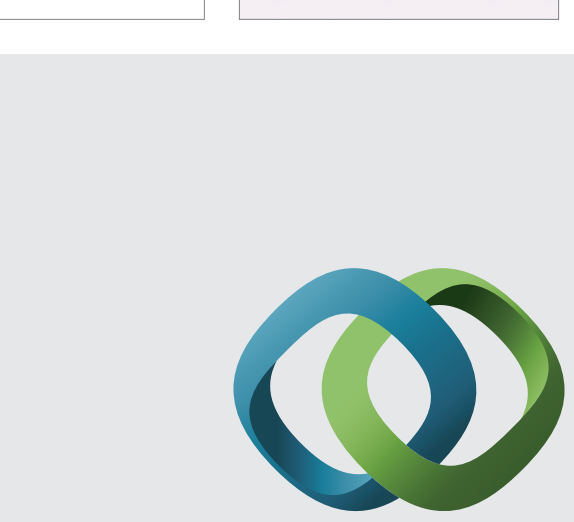

\section{Hindawi}

Submit your manuscripts at

http://www.hindawi.com
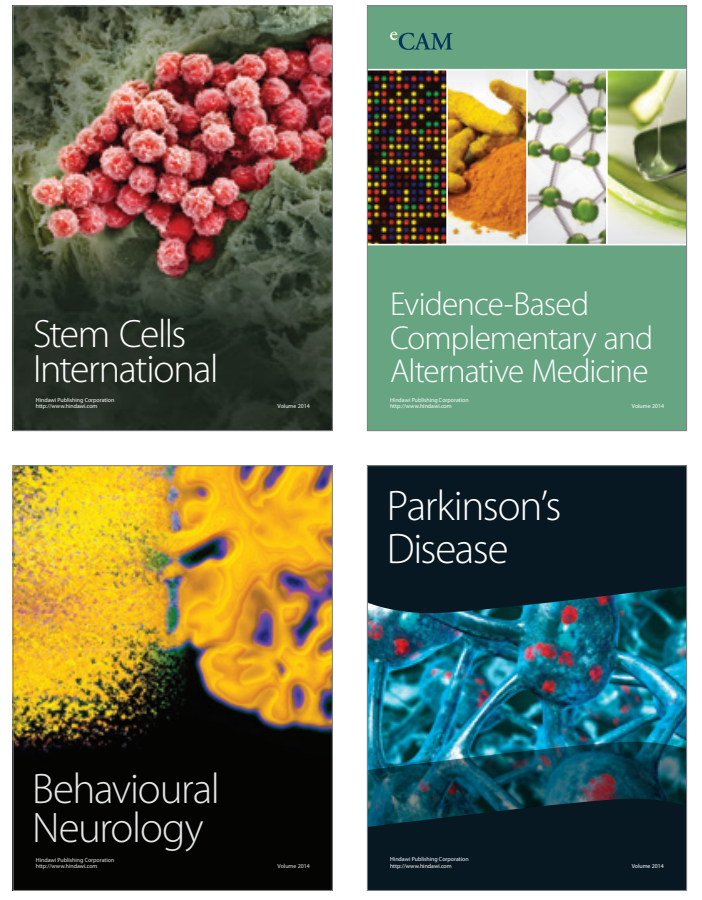
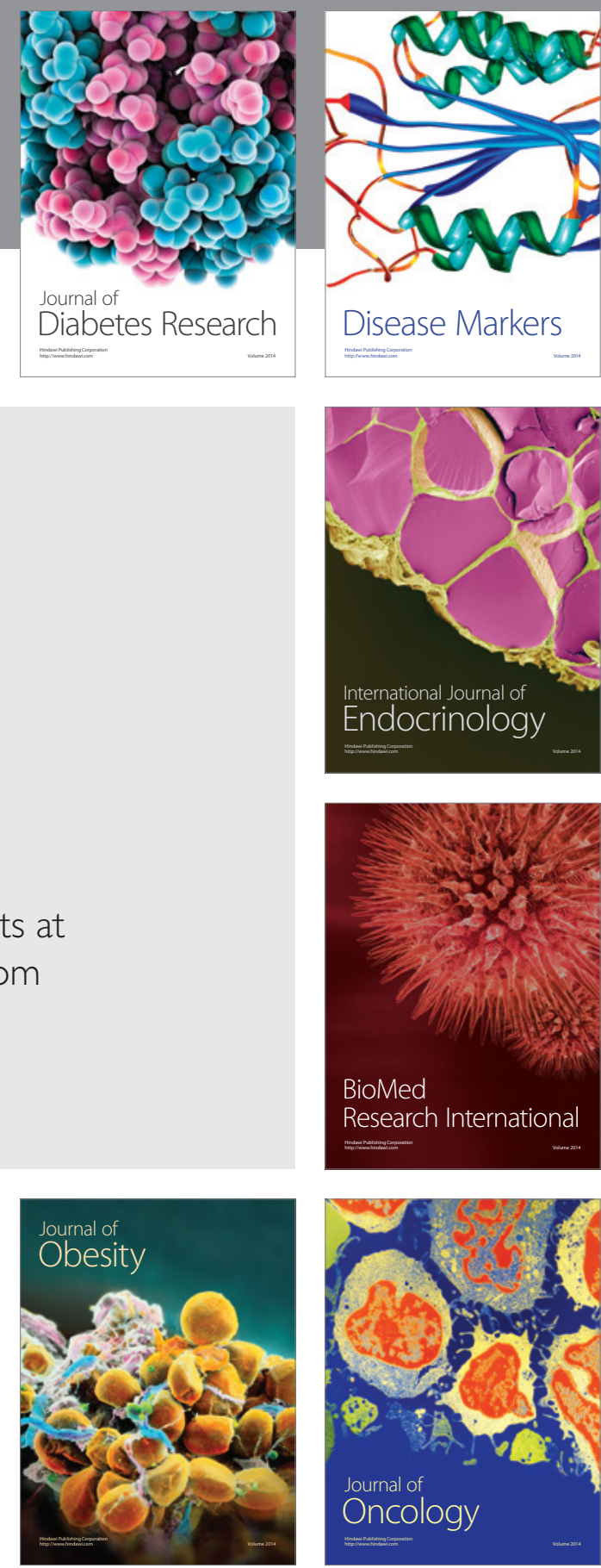

Disease Markers
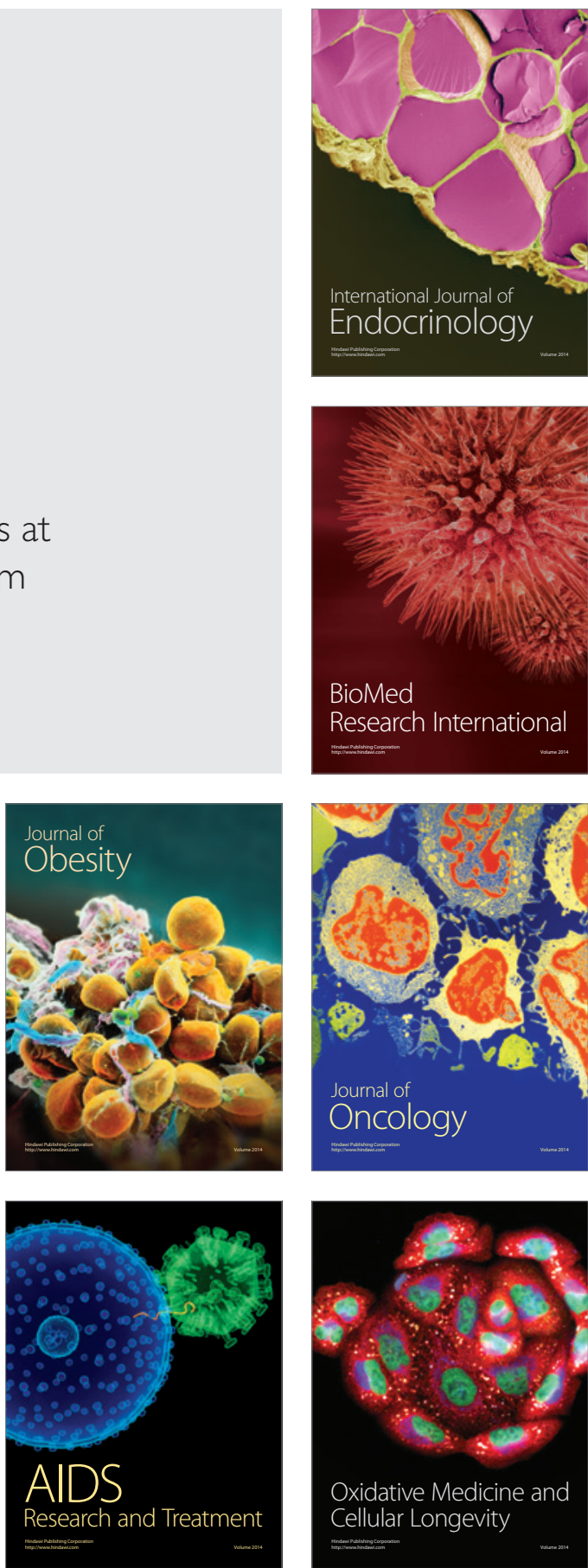